Open Access available at: http://journals.uct.ac.za/index.php/UR/index

DOI: 10.15641/ur-at-uct.v1i2.39

\title{
Levels of empathy in speech therapy and audiology undergraduate students training at the University of Cape Town
}

Christine Rogers ORCID: orcid.org/0000-0001-6624-0443

Zenzo Chakara (CHKZEN001@myuct.ac.za)

Romy Cohen (HNROM002@myuct.ac.za)

Co-authors: Kelsey Fourie, Danielle Gounder, Nyasha Makaruse

Corresponding author: Christine.Rogers@uct.ac.za

Faculty of Health Sciences, University of Cape Town

\begin{abstract}
Research has found empathy expressed by healthcare professionals to be beneficial to their relationship with patients, resulting in better patient outcomes as well as greater job satisfaction for clinicians. In line with the move towards patient-centred care, health science curricula have responded by including teaching of empathy, professional and communication skills to under-graduates. A body of research exists exploring empathy levels among Health Sciences students; however, little research has been conducted with students on the African continent. The current study sought to establish the levels of empathy of all Audiology and Speech-Language Pathology students enrolled at the University of Cape Town. The Jefferson Scale of Physician Empathy-Health Profession Student version (JSE-HPS) was used to assess levels of empathy among students across all years of study. Results were analysed in terms of year of study, gender and degree programme. Students entered both Programmes with a positive attitude towards empathy, and this was sustained throughout their training, although gender differences existed with male students expressing less orientation towards empathy. Implications are discussed in the light of a South African context.
\end{abstract}

Keywords: Empathy; Audiology; Speech-Language Pathology; students; Jefferson Scale of Physician Empathy-Health Profession Student version 


\section{Introduction}

Empathy can be defined as the ability to understand and interpret another person's feelings without affixing to them (Fields et al. 2011). Within the healthcare context, most researchers agree that empathy involves the health professional's appropriate understanding of and response to the patient's pain, suffering, experiences and perspectives without becoming overwhelmed; and relaying their intent to help the patient (Babar et al. 2013). Empathy as a cognitive process allows health professionals to objectively comprehend their patients' life and illness experiences (Gonullu \& Oztuna 2012).

\section{Benefits of empathy in health-care professionals and patients}

There is extensive research documenting benefits of empathy to both patients and their health care professionals (Wilson, Prescott \& Becket, 2012). In patients, cognitive benefits such as reduction in depression, anxiety and stress levels have been observed (Fields et al. 2011), as well as improved adherence to treatment regimens and better patient satisfaction (Wilson et al. 2012). Amongst healthcare professionals, higher levels of empathy have been reported to be closely related to improved clinical competence ratings, accurate diagnosis, better performance on history taking and higher ratings of job satisfaction (Farrelly, 2012; Tavakol, Dennick \& Tavakol, 2012; Tel, Mollaoglu, Polatkan \& Gunaltay, 2011). Importantly, high empathy levels have also been associated with a decrease in likelihood of litigation against health professionals (Fjortoft, Van Winkle \& Hojat 2011). In recent years, technological advancement has introduced equipment that can be operated without interaction between the practitioner and patient; therefore, an empathetic relationship is important in bridging the gap between patients and practitioners (Wilson et al. 2012, Gonullu \& Oztuna 2012). Research has shown that empathy - an acquired skill, rather than purely an innate ability - can be facilitated through teaching; therefore it is not surprising that empathy forms part of the patient-centred approach that is taught in the undergraduate curricula of many healthcare professions (Spiro 1992; Williams et al. 2014). 


\section{Research on empathy in allied health-care professional students}

Globally, much research on empathy has focused on medical and nursing students (Nunes, Williams, Sa \& Stevenson, 2011). Empathy is an important aspect in non-medical health professions and recommendations have been made to include training of the skill in allied health sciences curricula (Hojat et al. 2009). However, little empirical research has focussed on empathy levels in allied health students (Hojat et al. 2009). Research development has been slow because of confusions in defining and conceptualizing empathy; as well as a lack of agreement on an instrument that is both reliable and valid to measure empathy (Hojat et al. 2009). These same challenges have been observed within the African and South African context (Vallabh, 2011; Dehning et al. 2012). Scant research on empathy has been done within the South African context, with only one study having been conducted in undergraduate medical students in South Africa, which used the Jefferson Scale of Physician Empathy (Vallabh 2011). This lack of empathy research in South Africa suggests the need for further research to be conducted amongst allied Health Sciences students.

Differences in cultures, ethnicity, gender and sex stereotyping, choice of profession, religious beliefs and norms all contribute to how empathic engagement unfolds in different settings (Vallabh, 2008; Gonullu \& Oztuna, 2012). Therefore, it is important to study levels of empathy in a South African context because of its own unique social strata that influence how one shows empathy towards others. The current study attempts to start bridging the gap in current literature by focusing specifically on researching empathy levels in audiology and speech therapy students at a South African university (Boyle et al. 2010).

\section{Factors which influence levels of empathy}

It is possible that cultural traditions and norms may impact empathy levels. For example, high levels of empathy were observed in Japanese medical students; and this was attributed to Japan's collectivist society influence, science orientation and the inclusion of humanities courses in their medical curriculum (Wilson et al. 2012). African countries subscribe to the principle of Ubuntu, which Chaplin (2006) describes as the 
potential of being a good human, by striving to help people and putting the needs of the community and others above one's own. With the concept of Ubuntu we argue that we could expect similar results as South Africa is mostly considered a collectivist society (Gibson, Swartz \& Sandenbergh, 2002). Hence, the current study's exploration of empathy levels in the South African health sciences student population, particularly Speech Therapy and Audiology, will add to the available literature on empathy together with a contextual factor, which will in turn help to explain the observed results.

Existing research shows mixed results as to whether there is a gender difference in empathy (Rueckert, 2011). Studies using self-report measures to assess empathy have provided the most convincing evidence for gender differences in empathy (Rueckert 2011), with women scoring significantly higher than men, suggesting that women are empathic (Baron-Cohen \& Wheelwright 2004; Davis, 1980). In particular, it has been suggested that female occupational therapy, pharmacy and other healthcare students have higher empathy scores than their male counterparts (Fjortoft et al. 2011).

Currently there is little research comparing empathy levels amongst health professionals in developed versus developing countries. Recently, two studies were conducted using variations of the Jefferson Scale of Physician Empathy (JSPE), with one study being conducted in an emerging country and another in an industrialised country. The JSPE-Health Professions Student version and the JSPE-Student version have a score range of 20-140, with a higher score indicating a higher self-rated level of empathy (Chen, Lew, Hirschman \& Orlander, 2007; Williams et al. 2014). In a 2008 study conducted amongst 176 third year medical students at the Thomas Jefferson University in Philadelphia, in which the JSPE was used, the mean score for female students, which was higher than that of males, was 110.4 (Berg, Majdan, Berg, Veloski \& Hojat, 2011). In a 2008 study amongst 158 final year medical students at the University of Witwatersrand in South Africa, in which the JSPE-S was used, the mean score female students was 109 and was also higher than that of the male students (Vallabh, 2011). This comparison indicates no significant difference in empathy levels according to the JSPE when comparing students in developed and developing countries. 
From the early days of research in investigating the presence of empathy in students, studies have tracked the levels of empathy as the years of clinical training progress (Reynolds \& Scott, 2000; Sherman \& Cramer, 2005). Many reasons have been given as to why the general trend is that of a decrease in empathy amongst health sciences students (Hojat et al. 2009). It can be argued that students enter training to become health-care professionals because they are driven by an altruistic motive to help people, as enrolled health professions students have expressed high enthusiasm to help others and be service-orientated (Hafferty, 2002; Hojat, 2009). This could suggest high levels of empathy amongst these students and is indeed supported by the higher scores obtained by these students during their first years (Hojat, 2009; Sherman \& Cramer, 2005). After enrolment, however, the general observation in these studies has been that of a decline in empathy levels with each year spent in the clinical training setting (Reynolds \& Scott, 2000; Sherman \& Cramer, 2005). For example, junior dental students were shown to have higher empathy levels than their senior clinical peers who are in contact with patients (Sherman \& Cramer, 2005), with occupational therapy and psychology students also showing a decline in empathy levels (Reynolds \& Scott, 2000). Thus, although research is limited, there has been a common observation of a decline in empathy levels with clinical training amongst allied health students, medical and nursing students. Possible reasons for eroding levels of empathy include patient factors, the encouragement of detachment and a lack of appropriate modelling of empathic skills from teaching clinicians (Hojat et al. 2009).

\section{Methodology}

The aim of this study was to explore levels of empathy in undergraduate speech therapy and audiology students within and between different years of study at the University of Cape Town (UCT). A quantitative, descriptive cross-sectional survey design was used (Terre Blanche, Durrheim, \& Painter, 2012). The Jefferson Scale of Physician Empathy-Health Profession Student Version was used as an outcome measure as it is the most commonly used scale for measuring empathy and has been widely validated, and is specific to patient care (Berg et al. 2011; Vallabh, 2011). Specific objectives 
determined the impact of seniority in the training programmes, gender, and choice of future occupation.

\section{Participant description}

All consenting, adult speech therapy/audiology students at UCT were eligible for inclusion. Of a sampling frame of 300 registered students across four years of study, 250 participated, yielding a response rate of $83 \%$. Ages ranged from 18 to 34 years. A total of 31 participants' data were excluded due to incompletion. See Table 1 for a description of participants.

\begin{tabular}{|l|c|c|c|c|}
\hline $\begin{array}{l}\text { Profession \& } \\
\text { gender }\end{array}$ & \multicolumn{4}{|l}{ Year of study } \\
\hline & 1st & 2nd & 3rd & 4th \\
\hline $\begin{array}{l}\text { Speech Therapy } \\
\text { (male) }\end{array}$ & 3 & 1 & 0 & 0 \\
\hline $\begin{array}{l}\text { Speech Therapy } \\
\text { (female) }\end{array}$ & 47 & 33 & 29 & 22 \\
\hline $\begin{array}{l}\text { Speech Therapy } \\
\text { (gender } \\
\text { unknown) }\end{array}$ & 0 & 0 & 5 & 0 \\
\hline $\begin{array}{l}\text { Audiology (male) } \\
\text { Audiology } \\
\text { (female) }\end{array}$ & 41 & 21 & 18 & 14 \\
\hline $\begin{array}{l}\text { Audiology } \\
\text { (gender } \\
\text { unknown) }\end{array}$ & 0 & 4 & 2 & 2 \\
\hline $\begin{array}{l}\text { Profession } \\
\text { unknown }\end{array}$ & 0 & 0 & 0 & 65 \\
\hline $\begin{array}{l}\text { Total participants } \\
\text { Total possible } \\
\text { sample size (n } \\
\text { registered) }\end{array}$ & 85 & 61 & 60 & 44 \\
\hline $\begin{array}{l}\text { Response rate } \\
\text { per year (\%) }\end{array}$ & 99 & 80 & 86 & 65 \\
\hline
\end{tabular}

Table 1. Participant description arranged in year of study, profession and gender 


\section{Materials and procedure}

Permission to use students as research participants was granted from the University of Cape Town Faculty of Health Sciences Human Research Ethics Committee (HREF number 879/2014), year convenors and Department of Student Affairs. Students were recruited via a presentation describing the research to each class; and interested individuals were invited to stay after the class to complete the questionnaire. Careful attention was paid to the ethics of using student participants and there were no issues regarding possible incentives or coercion. Specifically, the principal investigator was not present which limited any possible power dynamic.

The Jefferson Scale of Physician Empathy-Health Profession Student version (JSPE-HPS) (Hojat et al. 2001), a self-report questionnaire, was used to measure empathy. This scale has been adapted to suit allied health students (Wilson et al. 2012) and is the most widely researched tool for assessment of student empathy (Vallabh, 2011). The scale contains 20 items which are scored on a Likert scale (where $1=$ strongly disagree and $7=$ strongly disagree). Ten positively worded items explore the skill of perspective taking whilst the rest have negative wording and are thus reverse scored (Fields et al. 2011). Negative wording controls for acquiescence (Hojat et al. 2009). Of the ten negatively worded items, eight are linked to compassionate care whilst two are linked to 'standing in the patient's shoes' (Vallabh, 2011). The Jefferson Scale has been found to be a robust measure for measuring empathy in allied health students (Wilson et al. 2012) and can be used as an evaluation measure where empathy education is being implemented (Wilson et al. 2012). It has been proven to be a psychometrically sound tool based on its test-retest reliability (Hojat, 2007), construct validity, internal validity and good internal consistency (Babar et al. 2013).

\section{Statistical analysis}

Descriptive statistics were used to determine levels of empathy in terms of mean and standard deviations (Terre Blanche et al. 2012). T-tests were used to compare: the overall scores of males and females and the overall scores of speech therapy and audiology students. Furthermore, t-tests were used to compare the scores of female and 
male students, as well as the scores of speech therapy and audiology students, within each year of study. A p-value of 0.05 was used to justify a claim of a statistically significant effect (Terre Blanche et al. 2012). Due to multiple comparisons being done, using t-tests, the risk of a Type 1 error occurring is increased, in which the null hypothesis is rejected when it is in fact true. In order to minimise the risk of a Type 1 error occurring, the Bonferroni Correction - a simple yet the most stringent method used to counteract the problems which occur when doing multiple comparisons of data - was applied in order to calculate the true p-value (Agilent Technologies Inc. 2005; Napierala 2012). Since $16 \mathrm{t}$ tests were conducted in the full analysis of this study's data, the true p-value was calculated to be 0.003125 rather than the traditional 0.05 .

\section{Results}

To establish the levels of empathy in each year group (first to fourth year), each participant's total score was determined and thereafter, the mean was calculated using the total score of each participant for that particular year. The minima and maxima are 20 and 140, respectively. The mean empathy scores across the years are presented in Figure 1.

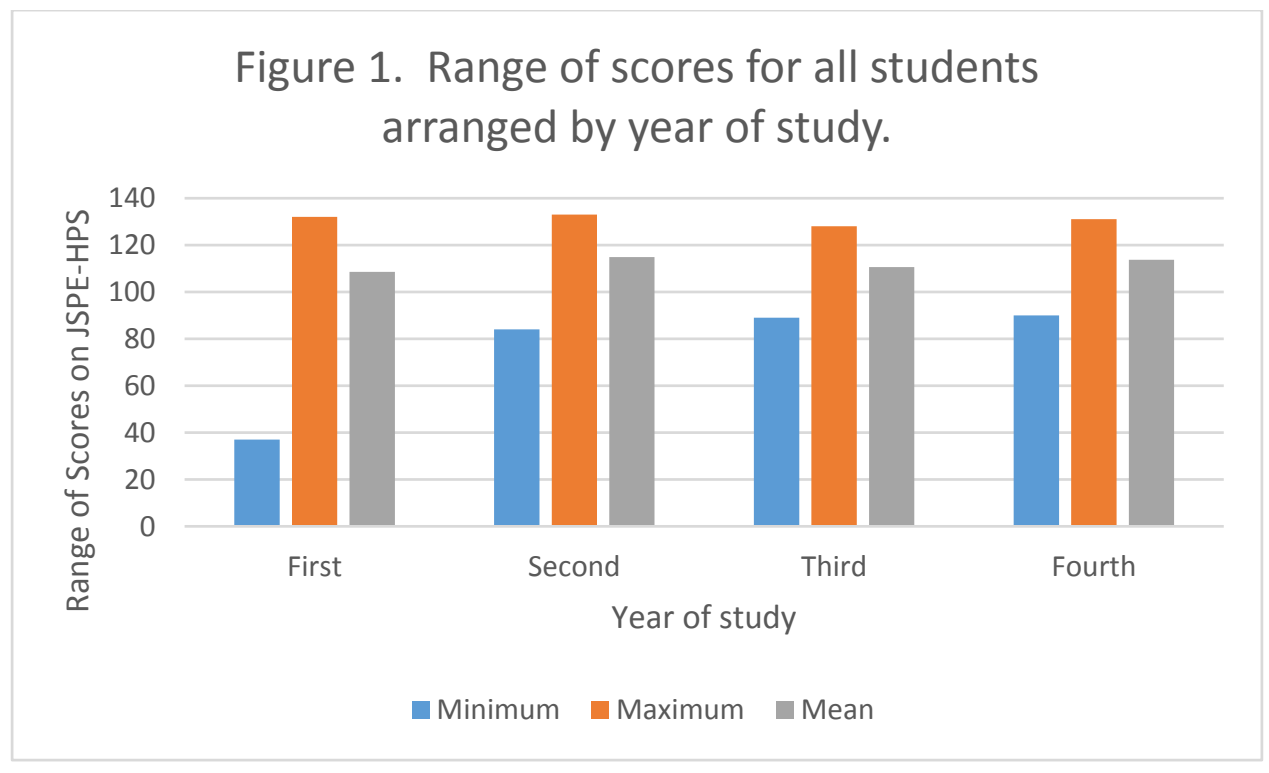


The overall mean of this study was 112 out of a possible 140, which is considered high. It should be noted that the first years had the widest range of scores with some exceptionally low scores (for example, 37). Means for each year were as follows: first, 108.6; second, 114.9; third, 110.6 and fourth, 113.7. Multiple t-tests ( $p=0.003125)$ were performed to further examine the difference between the means of each year group to explore the effect gender and progress through the programmes. The results found a significant difference between first and second year $(p=0.00)$ as well as first and fourth year $(p=0.00)$, with first years scoring significantly lower than their second and fourth year counterparts. A decline in empathy levels was shown in third year with a significant difference found between 3rd and 2 nd year $(p=0.00)$ as well as 3rd and 4 th year $(p=$ 0.04 ), with 3rd years scoring significantly lower than their 2 nd and 4 th year counterparts. T-tests further revealed no significant difference between 1st and 3rd year $(p=0.22)$ as well as 2 nd and 4 th year $(p=0.41)$.

Finally, a t-test revealed no significant difference between Speech Language Pathology and Audiology students $(p=0.06)$; thus Figure 1 shows the combined scores per year. A t-test $(p=0.003125)$ was performed comparing all the data from male and female students, overall and within each year. A significant difference between males and females was found, with males scoring lower than their female counterparts in first $(p=0.00)$ and second year $(p=0.00)$. However no significant difference was noted between males and females in third $(p=0.07)$ and fourth year $(p=0.01)$; during which there is more clinical practice.

To summarise, the results showed a score range of 37 to 133 across all participants; but were generally high. Overall, male students demonstrated significantly lower empathy levels than female students, whilst there was no significant difference between Speech Therapy and Audiology students. Empathy levels increased significantly between first and second year, decrease significantly between second and third year, and then increase significantly again between third and fourth year. 


\section{Discussion}

Empathy levels were high for first year students according to Hojat et al. (2002), who states that a higher score on the JSPE-HPS means that a student is more likely to behave in an empathic manner when engaging with patients. This suggests that novice Speech Language Pathology and Audiology students enter the programmes with good levels of empathy, presumably driven by high enthusiasm and orientation towards service, as was noted in previous research (Hojat, 2009). However, the lowest reported score, 37, obtained by a first year student, also suggests that a wide range of orientation toward empathy exists in this year. Therefore, it cannot be assumed that all students enter the Speech Language Pathology or Audiology programmes with innate levels of empathy. In addition, such a wide range of scores (37-132), would signal the need to develop these skills before students commence their clinical training. As the data collection was conducted during the first three weeks of enrolment, it is unlikely that the curriculum had shaped or influenced the first year students' empathy levels at this point.

Literature has cited mean scores of 115 (Brown et al. 2010), 124 (Hojat et al. 2010) and 117.2 (Fields et al. 2004) amongst Health Sciences students as being indicators of high empathy levels, indicating that students are capable of experiencing empathy for their patients. The results from the current study showed that, although the mean empathy scores were not as high as those found in previous studies, they were still comparable.

Similar to previous work, a significant difference in empathy scores was found between genders, with females scoring higher than males (Baron-Cohen \& Wheelwright, 2004; Davis, 1980; Rueckert \& Naybar, 2008). Various factors, including culture and socialization play an important role in the development of empathy (Baron-Cohen, 2005). In addition, many cultures require males and females to behave in certain ways; grooming individuals from a young age to comport themselves as such in order to be deemed socially acceptable (Rueckert, 2011). Many of these traditional sex-role stereotypes suggests that women are more nurturing and more likely to express a higher level of emotional empathy (Yu, Wang \& Liu, 2012). A further explanation of this difference could be that the participants of this study were aware that this study was exploring empathy as 
a function of gender. This awareness is likely to prompt responses influenced by an individual's identification with gender stereotypes, leading to women feeling the need to respond more empathically, whereas men feel they must respond less empathically (Rueckert, 2011).

Contrary to the observation of a decline in empathy levels with increasing seniority observed in previous research (Brown et al. 2002; Gonullu \& Oztuna, 2012), the current study showed an increase in empathy levels. Empathy scores were at their highest in second year for both Speech Language Pathology and Audiology students. In the senior years, the statistically significant difference in empathy levels between final and first year students with the former having the better scores; suggests empathy is a developing attitude in this sample at least.

The current study correctly predicted an increase in empathy with increasing seniority. The Speech Language Pathology and Audiology programmes at UCT contain Humanities courses such as Psychology in their curricula, which is cited as a contributing factor for high empathy levels (Gonullu \& Oztuna, 2012). The increase in empathy levels from first to second year may also be attributed to two multi-professional courses offered at the University of Cape Town's Faculty of Health Sciences (Olckers, Gibbs \& Duncan, 2007). These two multi-professional courses, namely "Becoming a Professional" and "Becoming a Health Professional" are taught in first year of study to all undergraduate Health Sciences students and aim to facilitate the growth of an integrated health professional (Olckers et al. 2007). Empathic practice in both courses aims to develop a new way for the first year students to think and view the different aspects of their respective professions (Olckers et al. 2007). This gradual building of an empathic foundation in both courses of the first year may suggest a possible reason for the observed increase from first year to second the participants.

As explored earlier, the context of student training is a society that subscribes to the concept of Ubuntu (Chaplin 2006). Thus, it is expected that empathy levels are, on average, high amongst Audiology and Speech Therapy students at UCT and that these 
levels tend to increase not only due to the orientation of the curriculum, but also as students come into contact with more patients in the latter years of their training. However, there is currently literature that points to young African adults beginning to abandon the concept Ubuntu in search of individual wealth which may have an impact on future healthcare professionals' levels of empathy, as they may grow up in societies where the spirit of Ubuntu is in decline (Broodryk 2006).

Unsurprisingly, the results demonstrated no significant difference between empathy scores of the two student-professions. As the admission protocol and requirements for both programmes are the same, it is likely that similar students are selected. Furthermore, after entering UCT, Speech Therapy and Audiology students study a number of similar courses, all of which reinforce the development of empathy. There is a strong multi-disciplinary connection between the two student-professions, which may also lead to development of similar approaches in handling situations that require empathy skills (Davis 1980).

\section{Implications of the study}

It is evident that students arrive at the University with a desirable orientation towards entering a caring profession. However, qualities such as empathy are not necessarily inherent. Its presence cannot be assumed in a student cohort as a wide range of scores was noted in first year. Based on the results, which show a significant increase in empathy levels from first year to second year, it is evident that the academic curriculum appears to be effective in increasing the empathy levels of undergraduate students in the Division of Communication Sciences and Disorders at the University of Cape Town. In spite of the demands of student clinical practice, there appeared not to be a systematic erosion of empathy during the more clinical years; suggesting that the values instilled at junior levels are upheld. Clinical educators in particular should be aware of gender-based differences in attitudes to empathy and work on these with their male students. 


\section{Limitations of the study}

Several factors resulting from the nature of this study and the manner in which data were obtained may have influenced the participants' responses. First, social desirability bias may have played a role in causing participants to respond in a way that they deem favourable or over-report attitudes that they believe are desirable (Connell, 1979 and Fowler, 1995; as cited in National Collaborating Centre for Primary Care, 2009). In this study, this risk of bias is more applicable to second, third and fourth year students, who have been exposed to empathy-based courses and modelling of empathetic behaviour during clinical training. Students are required to demonstrate an empathetic demeanour during clinical training as part of professional conduct, in contrast, first year students have not yet had this exposure and are therefore less likely to be influenced by these phenomena.

Furthermore, the Pygmalion effect may have played a role in the female participants' significantly higher reported empathy levels. The Pygmalion effect proposes that people respond or act in ways that conform to the expectations placed on them, such as social norms and, particularly in this study, gender roles (McLeod,1995). Sherman and Cramer (2005) noted that studies that solely rely on self-report measures of subjective experiences such as empathy may obtain inaccurate results, as students' reported orientation towards empathy and not their actual behaviour is recorded. The credibility of students' reported orientation towards empathy may be influenced by a variety of personal factors including honesty, understanding, introspective ability, self-perception and self-knowledge, all of which may affect the construct validity of the study (Hoskin, 2012; McDonald, 2008; Paulhus \& Vazire, 2009). In addition, frame-of-reference bias the fact that each participant may have a different perception of what it means to have a particular quality, such as empathy, as well as a different perception of the Likert scale ratings - is inherent in every participant (Beegle, Himelein \& Ravallion, 2012; West, 2014). This also influences their responses, as each participant will have a different standard of comparison regarding the constructs in the questionnaire (West, 2014). 


\section{Recommendations for future research}

The results of this study showed that overall, students at UCT enter with and maintain levels of empathy similar to other health professional students. Whilst these results show a pleasing orientation towards empathy, this research did not address which factors would promote increases in empathy. Courses within the programme promote empathy as a desirable characteristic of a health care professional. The expectation is that such values are demonstrated to students during the clinical experience. Further research needs to be conducted in order to explore whether empathetic behaviour is being modelled appropriately by clinical educators and other staff responsible for the training of Health Sciences students.

It is further recommended that a longitudinal design be used for further studies, in order to track the changing empathy levels of the first year participants who participated in this study from their first to fourth year of study. This will allow researchers to observe whether the changes in empathy reported in this study occur, as well as establish a sequence of effects, make causal inferences and provide information about individual change (Le Blanc, 2014). Furthermore, future studies should incorporate other divisions within the Health Sciences faculty and should extend to include other institutions offering Health Sciences programmes.

\section{Conclusion}

This study aimed to explore the levels of empathy amongst 250 undergraduate Audiology and Speech-Language Pathology students at the University of Cape Town, using the Jefferson Scale of Physician Empathy - Health Professional Student version. The results showed a significant difference between males and females, with females scoring significantly higher than males. Furthermore, the results showed that students across all four years scored highly on the JSE-HPS, with second and fourth year students scoring significantly higher than their first and third year counterparts. This contradicts the decrease in empathy levels that can be seen in existing research. Therefore, it is apparent that the current curriculum fosters empathy across the years. It is recommended that additional empathy-based courses and workshops be run for students throughout their degree programme, in order to ensure that empathetic attitudes are continually fostered 
Open Access available at: http://journals.uct.ac.za/index.php/UR/index

DOI: $10.15641 /$ ur-at-uct.v1i2.39

and that students are provided with opportunities to translate these attitudes into empathetic behaviour. 


\section{References}

Agilent Technologies Inc, 2005, Multiple testing corrections [Online handbook].

Retrieved on June 2, 2015 from

\section{https://www.chem.agilent.com/cag/bsp/sig/downloads/pdf/mtc.pdf}

Babar, M. G., Omar, H., Lim, L.P., Khan, S.A., Mitha, S., Ahmad, S. F. B., \& Hasan, S.S. (2013). An assessment of dental students' empathy levels in Malaysia. International Journal of Medical Education, 4, 223-229. doi: 10.5116/ijme.5259.4513.

Baron-Cohen, S., \& Wheelright, S. (2004). The empathy quotient: An investigation of Adults with Asperger syndrome or high-functioning autism and normal sex differences. Journal of Autism and Developmental Disorders, 34, 163-175.

Baron-Cohen, S. (2005). The essential difference: The male and female brain. In Phi KappaPhi Forum, 85, 23-27. Retrieved November 14, 2014 from https://www.questia.com/magazine/1G1-131332336/the-essential-difference-the ale-and-female-brain

Beegle, K., Himelein, K., \& Ravallion, M. (2012). Frame-of-reference bias in subjective welfare. Journal of Economic Behaviour and Organisation, 81, 556-570. doi: 10.1016/j.jebo.2011.07.020.

Berg, K., Majdan, J. F., Berg, D., Veloski, J., \& Hojat, M. (2011). A comparison of medical students' self-reported empathy with simulated patients' assessments of the students' empathy. Medical Teacher, 33, 388-391. doi:10.1097/ACM.0b013e3182224f1f.

Broodryk, J. (2006, October). Ubuntu: African life coping skills. Paper presented the Commonwealth Council for Educational Administration and Management (CCEAM), Pretoria, South Africa.

Brown, T., Williams, B., Boyle, M., Molloy, A., McKenna, L., Molloy, L., \& Lewis, B. (2010). Levels of empathy in undergraduate occupational therapy students. Occupational Therapy International, 17, 135-41. doi: 10.1002/oti.297. 
Chaplin, K. (2006). The Ubuntu spirit in African communities. Retrieved January 30, 2015 from

http://www.coe.int/t/dg4/cultureheritage/culture/Cities/Publication/BookCoE20Chaplin.pdf

Chen, D., Lew, R., Hirschman, W., \& Orlander, J. (2007). A cross-sectional measurement of medical student empathy. Journal of General Internal Medicine, 22, 1434 1438. doi:10.1007/s11606-007-0298-x.

Davis, M. H., (1980). A multidimensional approach to individual differences in empathy. Catalog of Selected Documents in Psychology, 10. Retrieved November 14, 2014 from http://www.eckerd.edu/academics/psychology/files/Davis 1980.pdf

Dehning, S., Girma, E., Gasperi, S., Meyer, S., Tesfaye, M., \& Siebeck, M. (2012). Comparative cross-sectional study of empathy among first year and final year medical students in Jimma University, Ethiopia: Steady state of the heart and opening of the eyes. BMC Medical Education, 12, 1-12. doi: 10.1186/1472 6920-12-34. Retrieved November 14, 2014 from http://www.biomedcentral.com/1472-6920/12/34

Farrelly, L. (2012). Measuring empathy in health care staff in relation to job satisfaction, job related affective well-being, gender, occupation and length of service. (Unpublished Bachelors final year project). Dublin Business School, Ireland. Retrieved November 14, 2014 from http://esource.dbs.ie/handle/10788/448

Fields, S.K., Mahan, P., Tillman, P., Harris, J., Maxwell, K., \& Hojat, M. (2011). Measuring empathy in health care profession students using the Jefferson Scale of Physician Empathy: Health Provider Student version. Journal of Interprofessional Care, 25, 287-293. doi: 10.3109/13561820.2011.566648.

Fjortoft, N., Van Winkle, L.J., \& Hojat, M. (2011). Measuring empathy in pharmacy students. American Journal of Pharmaceutical Education, 75, 1-6. doi: 10.5688/ajpe756109.

Gibson, K., Swartz, L., \& Sandenbergh, R. (2002). Counselling and Coping. Cape Town, South Africa: Oxford University Press.

Gonullu, I. \& Oztuna, D. (2012). A Turkish adaptation of the Student Version of the Jefferson Scale of Physician Empathy. Mamara Medical Journal, 25, 87-92. 
Hafferty, F.W. (2002). What medical students know about professionalism. The Mount Sinai Journal of Medicine, 69, 385-397.

Hojat, M. (2007). Empathy in patient care: Antecedents, development, measurement, And outcomes. Philadelphia, PA: Springer.

Hojat, M., Gonnella, J., Nasca, T.J., Mangione, S., Vergare, M., \& Magee, M. (2002). Physician empathy: Definition, components, measurement, and relationship to gender and speciality. American Journal of Psychiatry, 159, 1563-1569.

Hojat, M., Vergare, M. J., Maxwell, K., Brainard, G., Herrine, S.K., Isenberg, G. A., \& Gonnella, J.S. (2009). The devil is in the third year: a longitudinal study of erosion of empathy in medical school. Academic Medicine, 84, 1182-1191. doi: 10.1097/ACM.0b013e3181b17e55.

Hojat, M., Mangione, S., Nasca, T.J., Cohen, M.J., Gonnella, J.S., Erdmann, J.B., \& Magee, M. (2001). The Jefferson Scale of Physician Empathy: Development and preliminary psychometric data. Educational and Psychological Measurement, 61, 349-365.

Hoskin, R, 2012, March 3, The dangers of self-report [Online article]. Retrieved April 19, 2015 from http://www.sciencebrainwaves.com/uncategorized/the-dangers-of-self-report

Le Blanc, M. (2014). Developmental criminology: Thoughts on the past and insight for the future. In J. Morizot \& L. Kazemian (Eds.), The development of criminal and antisocial behaviour: Theory, research and practical applications (pp. 507-538). New York, NY: Springer.

McDonald, J. D. (2008). Measuring personality constructs: The advantages and disadvantages of self-reports, informant reports and behavioural assessments. Enquire, 1, 1-18.

McLeod, S. H. (1995). Pygmalion or Golem? Teacher affect and efficacy. College Compositions and Communications, 46, 369-386.

Napierala, M.A. (2012, April). What is the Bonferroni Correction? [Online article]. Retrieved May 2, 2015 from http://www.aaos.org/news/aaosnow/apr12/research7.asp 
National Collaborating Centre for Primary Care (UK), 2009, Medicines adherence: Involving patients in decision about prescribed medicines and supporting adherence. London, United Kingdom: Royal College of General Practitioners. Retrieved May 2, 2015 from https://www.nice.org.uk/guidance/cg76.

Nunes, P., Williams, S., Sa, B., \& Stevenson, K. (2011). A study of empathy decline in students from five health disciplines during their first year of training. International Journal of Medical Education, 2, 12-17. doi: 10.5116/ijme.4d47.ddb0.

Paulhus, D. L. \& Vazire, S. (2009). The self-report method. In R. W. Robins, R. C. Fraley, \& R. F. Krueger (Eds.), Handbook of research methods in personality psychology (pp.224-239). New York, NY: Guilford.

Reynolds, W. J., \& Scott, B. (2000). Do nurses and other professional helpers normally display much empathy? Journal of Advanced Nursing, 31, 226-234.

Rueckert, L. (2011). Gender differences in empathy. Psychology of Empathy, 221-234.

Rueckert, L., \& Naybar, N. (2008). Gender differences in empathy: The role of the right hemisphere. Brain and Cognition, 67, 162-167.

Sherman, J.J., \& Cramer, A. (2005). Measurement of changes in empathy during dental school. Journal of Dental Education, 69, 338-345.

Spiro, H. (1992). What is empathy and can it be taught? Annals of Internal Medicine, 116, 843-846. doi:10.7326/0003-4819-116-10-843.

Tavakol, S., Dennick, R., \& Tavakol, M. (2012). Medical students' understanding of empathy: A phenomenological study. Medical Education, 46, 306-316. doi: 10.1111/j.1365-2923.2011.04152.x.

Tel, H., Mollaoglu, M., Polatkan, R., \& Gunaltay, H. (2011). Empathic inclination and job satisfaction in the nurses working with the individuals with chronic diseases. European Psychiatry, 26, 403. doi: 10.1016/S0924-9338.

Terre Blanche, M.J., Durrheim, K., \& Painter, D., (Eds.). 2012, Research in practice: Applied methods for the social sciences. Cape Town, South Africa: Juta and Company Ltd.

Vallabh, K. (2011). Psychometrics of the student version of the Jefferson Scale of Physician Empathy (JSPE-S) in final-year medical students in Johannesburg in 2008. South African Journal of Bioethics and Law, 4, 63-68. doi: 10.7196/sajbl.164 
West, M.R., 2014, December 18, The limitations of self-report measures of non-cognitive skills. Retrieved April 19, 2015 from

http://www.brookings.edu/research/papers/2014/12/18-chalkboard-non-cognitivewest.

Williams, B., Brown, T., Boyle, M., \& Dousek, S., 2013, Psychometric testing of the Jefferson Scale of Empathy Health Profession Students' version with Australian paramedic students. Nursing \& Health Sciences, 15, 45-50. doi: 10.1111/j.14422018.2012.00719.x.

Williams, B., Brown, T., Boyle, M., McKenna, L., Palermo, C., \& Etherington, J. (2014). Levels of empathy in undergraduate emergency health, nursing, and midwifery students: A longitudinal study. Advances in Medical Education and Practice, 5, 299-306. doi:10.2147/AMEP.S66681.

Williams, B., Brown, T., McKenna, L., Boyle, M. J., Palermo, C., Nestel, D., Brightwell, R., McCall, L., \& Russo, V. (2014). Empathy levels among health professional students: a cross-sectional study at two universities in Australia. Advances in Medical Education and Practice, 5, 107-113. doi: 10.2147/AMEP.S57569.

Wilson, S.E., Prescott, J., \& Becket, G. (2012). Empathy levels in first-and third-year students in health and non-health disciplines. American Journal of Pharmaceutical Education, 76, 1-4. doi: 10.5688/ajpe76224.

Yu, G., Wang, Y., \& Liu, C., 2012, Improving public service quality from a developmental perspective: Empathy, attachment, and gender differences. Public Personnel Management, 41, 9-20. doi:10.1177/009102601204100502. 